\title{
Efeitos do vento solar na magnetosfera terrestre: uma abordagem didática dos cinturões de Van Allen
}

Effects of the solar wind on the Earth's magnetosphere: a pedagogic approach to the Van Allen belts

\author{
R. S. Dutra*1@, D. S. R. Ferreira ${ }^{1}$, A. S. M. Gonçalves ${ }^{1}$, G. M. Carvalho ${ }^{1}$
}

${ }^{1}$ Instituto Federal do Rio de Janeiro, Laboratório de Instrumentação e Simulação Computacional, Paracambi, RJ, Brasil

Recebido em 06 de julho de 2019. Revisado em 24 de outubro de 2019. Aceito em 04 de novembro de 2019

\begin{abstract}
Neste trabalho realizamos uma abordagem didática de aspectos relacionados ao geomagnetismo e como esses aspectos podem ser influenciados pela ação do vento solar. A carência de textos didáticos que promovam explicações em termos de conceitos de física básica foi a principal motivação para esse trabalho. Iniciamos com uma discussão histórica sobre o estudo do magnetismo que culminou na conhecida teoria do dínamo para explicar a origem do campo magnético terrestre, na qual mostramos a possiblidade de abordar este assunto em cursos de eletricidade e magnetismo. Apresentamos também dois cenários para a magnetosfera terrestre, com o intuito de modelar a interação do vento solar com a magnetosfera, em que representamos tal interação por meio da superposição dos campos de dois dipolos magnéticos. A seguir mostramos a possiblidade de discutir o movimento das cargas provenientes do vento solar ao redor da Terra, na região da magnetosfera, utilizando a segunda lei de Newton e a força de Lorentz, discutindo assim a formação de anéis de cargas em movimento e dos cinturões de Van Allen. Finalmente apresentamos um simples argumento, baseado no modelo apresentado, para a deformação da magnetosfera, para explicar a limitação na quantidade de anéis e cinturões que envolvem o nosso planeta.

Palavras-chave: Geomagnetismo; Cinturões de Van Allen; Vento solar.
\end{abstract}

In this work we perform a pedagogic approach to aspects related to geomagnetism and how they can be influenced by the action of the solar wind. The lack of didactic textbooks that promote explanations in terms of basic physics concepts is the main motivation for this work. We begin with a historical discussion about the study of the magnetism that culminated in the well-known dynamo theory which explains the origin of the Earth's magnetic field, where we show the possibility to treat this subject in classes of electricity and magnetism. We also present two scenarios for the Earth's magnetosphere, in order to model the interaction of the solar wind with the magnetosphere, where we represent such action by superposing the fields of two magnetic dipoles. After we show the possibility of discussing the movement of charges coming from the solar wind around the Earth, in the region of the magnetosphere, using Newton's second law and the Lorentz force, where we discuss the formation of moving charged rings and the well known Van Allen belts. Finally, we present a simple argument based on the presented model, for the deformation of the magnetosphere, to explain the limitation in the amount of rings and belts that surround our planet.

Keywords: Geomagnetism; Van Allen belts; Solar wind.

\section{Introdução}

Dados históricos mostram que o estudo documentado do geomagnetismo teve início no século XIII a partir de uma carta, escrita pelo estudioso francês Pedro Peregrino, que ficou conhecida como a Epistola de Magnete [1], sendo considerado por muitos, o primeiro tratado de física experimental, precedendo em mais de três séculos as leis da mecânica newtoniana. Neste tratado, leis da atração e repulsão magnética são descritas, bem como o funcionamento de bússolas. Todavia foi o médico inglês William Gilbert quem fez o primeiro estudo sistemático dos fenômenos magnéticos, inaugurando uma nova metodologia no estudo do magnetismo, por meio do livro

*Endereço de correspondência: rafael.dutra@ifrj.edu.br
De Magnete 2], publicado em 1600. Com o passar dos séculos as bússolas passaram a ser cada vez mais utilizadas, principalmente no período das grandes navegações, séculos XV e XVI, quando grandes descobertas foram feitas, tendo sido um importante instrumento de navegação nessa época. Ao lado das aplicações do magnetismo terrestre nas navegações (até aquela época ainda era um mistério a sua origem) estava também o esforço dos cientistas na tentativa de compreendê-lo. Por volta de 1820 o físico dinamarquês Hans Christian Oersted realizou um experimento que desvendaria a origem do magnetismo, estabelecendo uma relação íntima de causa e efeito entre eletricidade e magnetismo, quando o mesmo verificou a deflexão da agulha de uma bússola por uma corrente elétrica que passava em um fio próximo à bússola [3]. Esse experimento abriu portas para a compreensão de 
diversos fenômenos elétricos e magnéticos, fundando assim a ciência do eletromagnetismo, na qual o campo geomagnético se configura como uma das manifestações eletromagnéticas de maior escala.

As leis do eletromagnetismo estruturadas em uma sólida teoria por James Clerk Maxwell, por volta de 1873, impulsionaram o estudo do geomagnetismo tanto do ponto de vista teórico quanto experimental, fazendo com que mais à frente fosse proposto um mecanismo para explicar a origem do campo magnético terrestre, que ficou conhecido como a teoria do dínamo [4], baseada na presença de um fluido condutor em movimento de rotação e convecção, presente na região do núcleo externo da Terra, sendo esta teoria a mais aceita até os dias atuais. Essa teoria proporciona um modelo didático no qual um fluido condutor composto de ferro líquido gira produzindo um campo magnético, análogo ao que acontece com uma corrente circulando em um anel. Como uma aproximação de ordem zero podemos pensar no campo magnético terrestre como sendo o de um dipolo magnético, sendo tal modelo sempre exposto nos livros didáticos.

Por sua vez, a Terra é protegida de diversas partículas carregadas provenientes dos ventos solares e raios cósmicos, em que tal proteção se deve à magnetosfera [5]. Observações experimentais da magnetosfera terrestre ao longo dos anos, através de medidas do vento solar no espaço interplanetário [6, 7], nos permitem modelar a mesma em termos da resultante da interação do campo dipolar da Terra com o vento solar 8, 9] (ver Figura 1), que carrega consigo o campo magnético interplanetário. O vento solar por sua vez é composto de partículas carregadas [10, em algumas ocasiões bastante energéticas, que graças a existência da magnetosfera, não atingem a superfície da Terra [11 13]. As partículas carregadas de altas energias ao entrarem na magnetosfera e interagirem com o campo geomagnético podem vir a ser capturadas

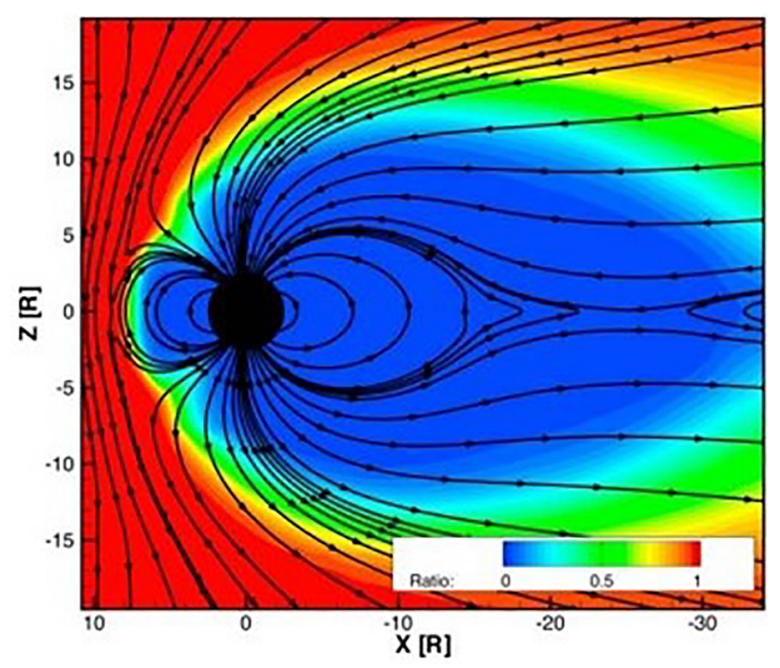

Figura 1: llustração retirada da referência [8], simula a interação entre o vento solar e a magnetosfera por meio da teoria magnetohidrodinâmica (MHD). e aprisionadas, realizando uma espécie de movimento confinado em torno da Terra, de tal modo que se pensarmos em milhões dessas partículas carregadas chegando, podem vir à formar cinturões dessas partículas envolvendo a Terra, dando origem aos famosos cinturões de Van Allen, descobertos em 1958, pelo físico James Van Allen 14, 15.

Como resultado, os cinturões defletem partículas energéticas provenientes do vento solar e raios cósmicos, protegendo a atmosfera terrestre e os satélites geoestacionários de uma eventual destruição. Para que não haja confusão é importante diferenciarmos a corrente de partículas que circula o nosso planeta, provenientes do vento solar, dando origem aos cinturões de radiação, dos conhecidos "ring currents". Os "ring currents" sempre existem em decorrêcia do movimento das partículas na presença de campos magnéticos com curvatura e divergência, sendo os mesmos intensificados nos eventos de tempestades magnéticas quando o acoplamento entre o vento solar e a magnetosfera se torna forte e prolongado o suficiente 16 . Apesar dos "radiation belts" e "ring currents" residirem na magnetosfera interna, ambos são populados de formas diferentes [17]. Os livros didáticos de física, tanto de ensino superior [18] quanto de ensino médio, ainda exploram e discutem de maneira incipiente a formação dos cinturões. Neste trabalho iremos apresentar um estudo da dinâmica de partículas carregadas que interagem com o campo magnético terrestre, com o intuito de apresentar, de maneira didática, a dinâmica de formação dos cinturões de Van Allen. Na segunda seção iremos apresentar e discutir a ideia de modelar o campo magnético da Terra em dois cenários: primeiro iremos considerar a aproximação de um único dipolo para o campo magnético da Terra e depois iremos apresentar uma possível maneira de simular a deformação do campo magnético terrestre causada pelo vento solar, considerando o modelo resultante da soma dos campos de dois dipolos separados por uma determinada distância, quando discutiremos a relevância e limite de validade de ambos os modelos. Na terceira seção iremos apresentar resultados numéricos para as trajetórias das partículas nos dois diferentes cenários, onde também discutiremos a formação dos cinturões em ambos os modelos. Finalizaremos com as conclusões e considerações finais na quarta seção.

\section{Dois Cenários para a Magnetosfera}

Nos livros didáticos de física é comum a representação do campo magnético terrestre como possuindo o formato das linhas de campo de um ímã, descrito pelo modelo de um dipolo magnético [19], podendo este ser visto como uma aproximação de ordem zero para a magnetosfera terrestre. Apesar da simplicidade do modelo, o mesmo pode ser utilizado como um ótimo laboratório de introdução ao geomagnetismo, para estudantes de ensino superior nos anos iniciais dos cursos de ciências e engenharias. Uma ótima alternativa para introduzir a aproximação dipolar 
para o campo magnético terrestre para ser aplicada nas aulas sobre magnetostática, nos cursos básicos de eletricidade e magnetismo, em que é abordado o problema de determinar o campo magnético gerado por um anel de raio $R$, percorrido por uma corrente $I$, em um ponto genérico do espaço, em que a corrente circulante no anel promove uma figura pictórica do movimento do fluido condutor na região do núcleo terrestre.

O campo magnético de um dipolo 20], associado a um anel centrado na origem (centro da Terra), de acordo com a Figura 2, é dado por

$$
\mathbf{B}(\mathbf{r})=\frac{\mu_{0}}{4 \pi}\left[\frac{3 \hat{\mathbf{r}}(\mathbf{m} \cdot \hat{\mathbf{r}})-\mathbf{m})}{r^{3}}\right],
$$

sendo $\mathbf{m}=\pi R^{2} I \hat{\mathbf{z}}$, o vetor momento de dipolo magnético, cujo módulo para o caso da Terra pode ser estimado em $8.10^{22} \mathrm{~J} / T$ [21], por meio de medidas do campo magnético, e $\mu_{0}=4 \pi \cdot 10^{-7} T . m / A$ a permeabilidade magnética do vácuo. Vale chamar a atenção para o fato de que, neste modelo dipolar, a magnitude do momento de dipolo magnético da Terra é proporcional a corrente elétrica $I$, associada ao movimento do fluido condutor, na região do núcleo externo, cuja extensão média é representada pelo raio $R$ do anel. Para os valores do momento de dipolo magnético terrestre e do raio médio do núcleo externo, estimado em $R \simeq 3481 \mathrm{~km}$ [22,23], estimamos uma corrente $I \simeq 2,1.10^{9} \mathrm{~A}$ associada ao fluido condutor circulante.

Na Figura 3, comumente vista em textos didáticos [19, vemos as linhas de campo magnético da Terra interpretadas em termos do modelo dipolar, por meio da equação (1). De acordo com este modelo a magnetosfera terrestre possui uma simetria azimutal de rotação em torno do eixo vertical $z$, além de se estender por todo o espaço, sendo este o exemplo clássico do campo magnético de um ímã. Contudo é sabido que a magnetosfera terrestre não possui simetria azimutal de rotação, sendo a mesma quebrada pela deformação das linhas de campo, em decorrência das partículas carregas do plasma solar, provenientes do vento solar, que perturbam a magnetosfera. Além de quebrar a simetria azimutal, o vento solar limita a magnetosfera por meio da magnetopausa, fronteira que delimita as regiões do plasma solar e da magnetosfera [24]. Um artifício simples, com o intuito de modelar a perturbação

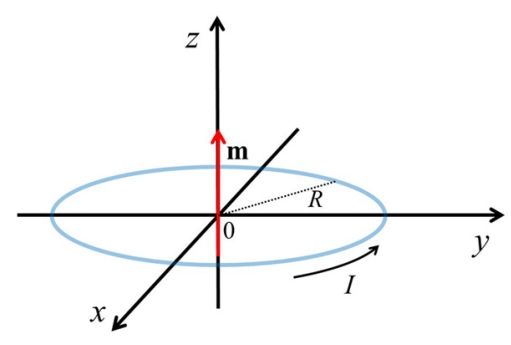

Figura 2: Anel de raio $R$, simulando a extensão média do núcleo externo da Terra, sendo percorrido por uma corrente $I$, simulando a corrente proveniente do movimento do fluido condutor.

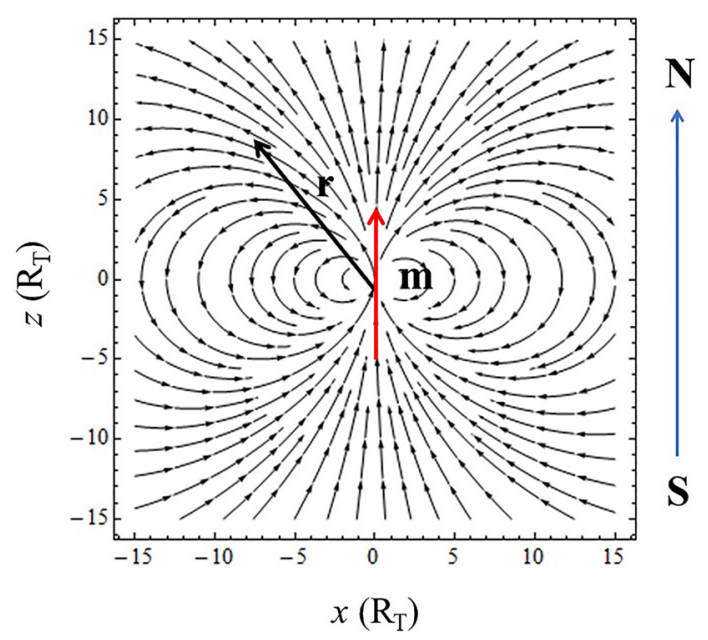

Figura 3: Vista lateral, no plano meridional $(x z)$, das linhas de campo magnético terrestre no modelo de um único dipolo. Seta azul: indica os polos norte e sul magnéticos da Terra. Seta vermelha: momento de dipolo magnético da Terra. Seta preta: um exemplo de vetor posição $\mathbf{r}(x, y, z)$ medido em relação ao centro da Terra. As dimensões dos eixos estão em unidades do raio da Terra, considerando-se $\mu_{0}=4 \pi \cdot 10^{-7} T$. $m / A$ (permeabilidade magnética do vácuo) e $m=8.10^{22} \mathrm{~J} / \mathrm{T}$ (módulo do vetor momento de dipolo magnético da Terra).

da magnestosfera pelo vento solar 25], consiste em considerar a superposição dos campos magnéticos de dois dipolos separados por uma distância $d$, como exposto na Figura 4. A metodologia consiste em superpor o campo $\mathbf{B}^{\prime}(\mathbf{r})$ de um outro dipolo localizado à direita da Terra, de momento de dipolo magnético $\mathbf{M}$, ao campo magnético $\mathbf{B}(\mathbf{r})$ da Terra, representado pela equação (1), com

$$
\mathbf{B}_{\text {total }}(x, y, z)=\mathbf{B}(x, y, z)+\mathbf{B}^{\prime}(x+d, y, z)
$$

representando o campo magnético resultante em todo o espaço. Para explicitar a dependência do campo magné-

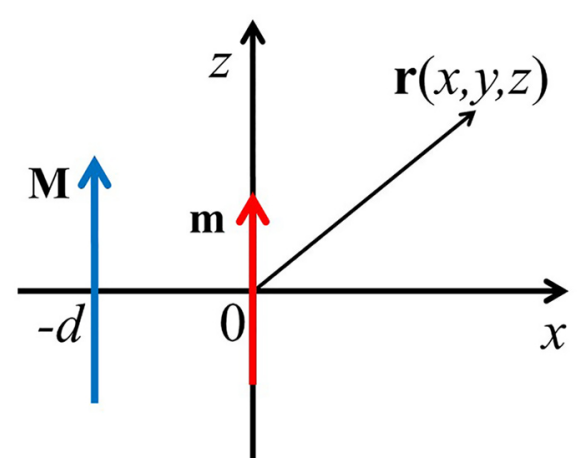

Figura 4: Vista lateral, no plano meridional $(x z)$, de dois dipolos separados de uma distância lateral $d$. Seta vermelha: vetor momento de dipolo magnético da Terra $\mathbf{m}$. Seta azul: vetor momento de dipolo magnético $\mathbf{M}$ adicionado para perturbar o campo magnético da Terra, simulando o efeito do vento solar. Seta preta: um exemplo de vetor posição $\mathbf{r}(x, y, z)$ medido em relação ao centro da Terra. 
tico total com o fator $n=M / m$, apresentamos, a título de ilustração, a componente $z$ do mesmo:

$$
\begin{gathered}
\mathbf{B}_{z, \text { total }}(x, y, z)=\frac{\mu_{0} m}{4 \pi}\left\{\frac{2 z^{2}-x^{2}-y^{2}}{\left(x^{2}+y^{2}+z^{2}\right)^{5 / 2}}+\right. \\
\left.\frac{n\left(2 z^{2}-(x+d)^{2}-y^{2}\right)}{\left((x+d)^{2}+y^{2}+z^{2}\right)^{5 / 2}}\right\}
\end{gathered}
$$

A localização do dipolo $\mathbf{M}$ depende da posição do Sol em relação à Terra, tal que essa escolha é arbitrária e sem perda de generalidade, e portanto, decidimos fazê-la com o mesmo localizado à direita da Terra. Os parâmetros $d$, distância entre os dipolos, e a razão entre os módulos dos momentos de dipolo $n=M / m$, controlam a intensidade da deformação das linhas de campo, simulando dessa forma a intensidade do vento solar. A posição escolhida para o dipolo imagem define o lado diurno da magnetosfera, uma vez que observações da magnetosfera indicam ausência de componente perpendicular do campo magnético na fronteira definida pela magnetopausa [26]. Na Figura 5 tomamos a distância entre os dipolos igual a vinte vezes o raio da Terra $\left(R_{T}=6371 \mathrm{~km}\right)$, ou seja, $d=20 R_{T}$. Na Figura 5 (a) ao considerarmos $n=1$ (dipolos de mesma intensidade), a fronteira conhecida como magnetopausa, que limita as regiões da magnetosfera terrestre e do plasma advindo do vento solar, é descrita por um plano vertical na metade da distância que une os dipolos, $x=-d / 2=-10 R_{T}$, que na vista lateral no plano meridional $x z$ é representada pela linha reta vermelha vertical. Esta figura se assemelha bastante com a figura mostrada por Chapman e Bartels [27]. Chapman naquela discussão usou o método da imagem para determinar os limites externos do que hoje conhecemos como magnetosfera terrestre. Portanto, os resultados de Chapman podem ser interpretados como o resultado da interação de um fraco vento solar com a magnetosfera. Isso faz sentido porque a existência do vento solar não era experimentalmente conhecida, nem teoricamente aceita, na década de 40. Já na Figura 5 (b) aumentamos a razão entre os momentos de dipolo para $n=20$ (dipolo externo 20 vezes mais intenso que o da Terra), o que leva à deformação da magnetosfera e consequentemente da magnetopausa, representada pela curva vermelha, levando a uma magnetosfera mais realista, similar às figuras comumente apresentadas em reportagens e em artigos científicos quando o tema envolve discussões sobre a interação do vento solar com a magnetosfera terrestre, obtidas por meio de simulações de magnetohidrodinâmica (MHD) [8 28,29 (ver Figura 1). Os resultados para o modelo de dois dipolos mostram que a magnetopausa se move na direção da Terra até $x=5 R_{T}$, em relação ao seu centro, como resultado da interação de um vento solar intenso com a magnetosfera, como representado na Figura 5(b). Um deslocamento da magnetosfera, desta magnitude, traria sérios riscos a satélites geoestacionários que orbitam a Terra em $x=6.6 R_{T}$ [30. Na próxima seção discutiremos o movimento de partículas carregadas dentro dos dois cenários apresentados nessa seção para a magnetosfera, na qual mostraremos uma maneira de abordar a formação dos conhecidos cinturões de Van Allen.

\section{Formação dos Cinturões}

O plasma solar advindo do vento solar é em sua grande parte composta de prótons, elétrons e íons pesados. Uma dada partícula carregada de carga $q$ e massa $m_{p}$ ao entrar (a)

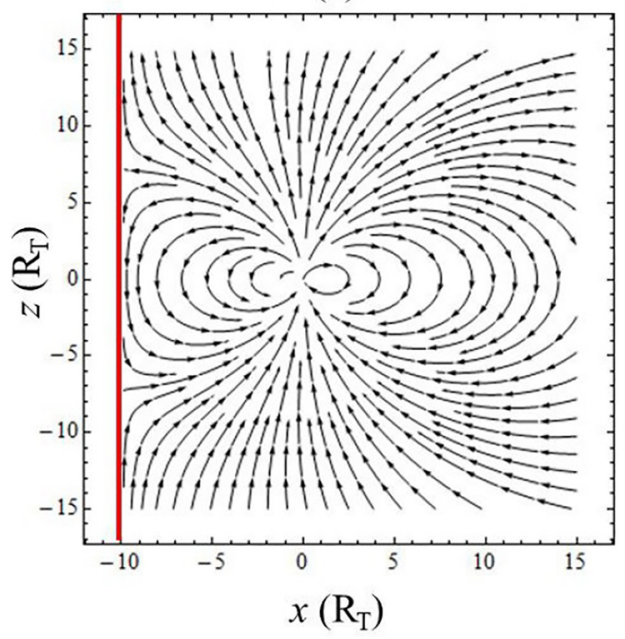

(b)

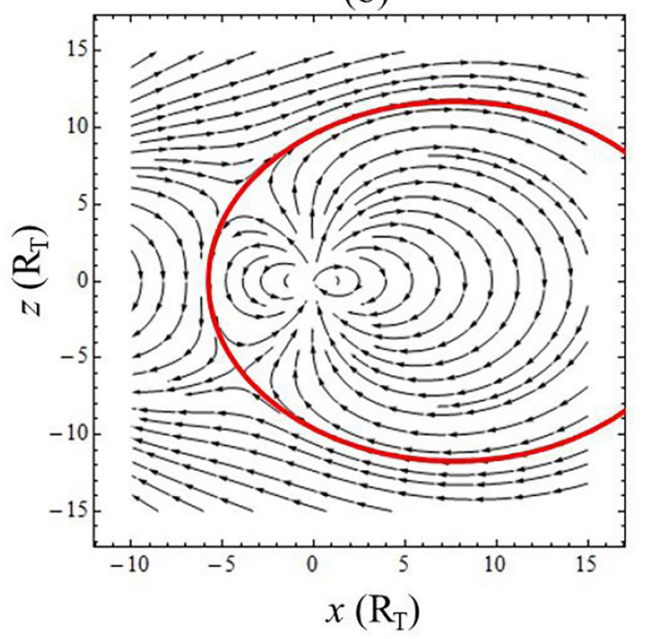

Figura 5: Vista lateral, no plano meridional $(x z)$, da magnetosfera terrestre deformada pelo vento solar. As linhas vermelhas, em ambas as figuras, representam a magnetopausa. (a) Situação em que os dipolos possuem o mesmo momento de dipolo, $n=1$. (b) Situação em que o dipolo adicional é vinte vezes mais intenso que o da Terra, $n=20$. As dimensões dos eixos estão em unidades do raio da Terra. 
no campo magnético da Terra passa a sofrer a ação da força de Lorentz de tal modo que a dinâmica da mesma, em uma primeira aproximação, pode ser descrita por

$$
q \mathbf{v} \times \mathbf{B}=m_{p} \frac{d^{2} \mathbf{r}}{d t^{2}} .
$$

Nesta descrição iremos desconsiderar a interação elétrica entre as cargas, tratando-as independentemente, como também iremos considerar a dinâmica newtoniana para descrever o movimento, não levando em conta efeitos relativísticos 31]. Apesar de parecer contraditório utilizar a mecânica newtoniana para descrever as partículas carregadas de altas energias do vento solar, que possuem energias típicas da ordem de $M e V$, veremos mais adiante que tal aproximação será suficiente para capturarmos os aspectos globais dos movimentos dessas partículas em torno da Terra, sendo esse um outro aspecto didático da exposição se pensarmos em abordar este assunto em uma classe introdutória de eletricidade e magnetismo, em cursos superiores em ciências e engenharias, em que os estudantes ainda não tiveram contato com os conceitos e descrição matemática da mecânica relativística. A equação (4) dá origem a três equações diferenciais não lineares e acopladas, uma para cada coordenada, de tal modo que utilizamos o software Mathematica para solucioná-las numericamente. Consideramos em nossas simulações prótons de massa $m_{p}=1,67.10^{-27} \mathrm{~kg}$ e carga $q=1,6.10^{-19} C \mathrm{com}$ energia inicial típica $E=1,6.10^{-12} J$, que será conservadada em decorrência do trabalho nulo realizado pela força de Lorentz:

$$
d W=q(\mathbf{v} \times \mathbf{B}) \cdot d \mathbf{l}=0,
$$

pois o elemento de linha $d \mathbf{l}$ é paralelo ao vetor velocidade $\mathbf{v}$, e consequentemente perpendicular à força $q \mathbf{v} \times \mathbf{B} \mathrm{em}$ cada ponto da trajetória. Portanto o módulo do vetor velocidade da carga, em qualquer ponto da trajetória, será constante e expresso por $v=\sqrt{2 E / m_{p}}$. Consequentemente, as trajetórias passam a depender fortemente do ângulo formado pela velocidade inicial da partícula e do vetor campo magnético em um dado ponto do espaço. Em nossas simulações consideramos o vetor velocidade inicial expresso por $\mathbf{v}_{0}=v \cos \theta \hat{\mathbf{x}}+v \sin \theta \hat{\mathbf{z}}$, paralelo ao plano $x z$, formando um ângulo $\theta$ com a direção $x$. Na Figura 6 (a) selecionamos prótons com velocidades iniciais na direção do equador $\theta=0$, que ao interagirem com o campo magnétco passam a executar um movimento circular, combinado com um movimento cicloidal, em torno da Terra, formando anéis de correntes. Os anéis foram formados considerando posições iniciais diferentes ao longo da direção $x$, durante o intervalo de 200 segundos. Por outro lado, como exposto na Figura 6 (b), o vento solar limita a quantidade de anéis de correntes formados pelos prótons com energias da ordem de $10 \mathrm{MeV}$. No cenário da Figura 6 (b) temos considerado dois dipolos separados por uma distância $d=20 R_{T}$, cuja razão entre os momentos de dipolo vale $n=20$, para simular a ação do vento solar sobre a magnetosfera terrestre. Pode-se observar, na Figura 6(b), que ao se considerar o modelo de dois dipolos o vento solar, simulado pela presença do segundo dipolo, varre os dois anéis mais externos existentes no modelo de um único dipolo Figura 6(a), fazendo portanto com que no modelo de dipolo duplo tenha-se somente os dois anéis mais internos.

Quando o ângulo formado entre a velocidade da carga e o campo magnético for diferente de 90, existe a possibilidade dos prótons executarem um tipo de movimento confinado, especial, em torno da Terra. As cargas descrevem um movimento helicoidal em torno das linhas de campo, sofrendo reflexões (inversões nos movimentos) nas regiões onde o campo magnético é mais intenso, formando cinturões de partículas carregadas envolvendo a Terra, os famosos cinturões de Van Allen. Na referência [32], tal movimento confinado das cargas, que origina
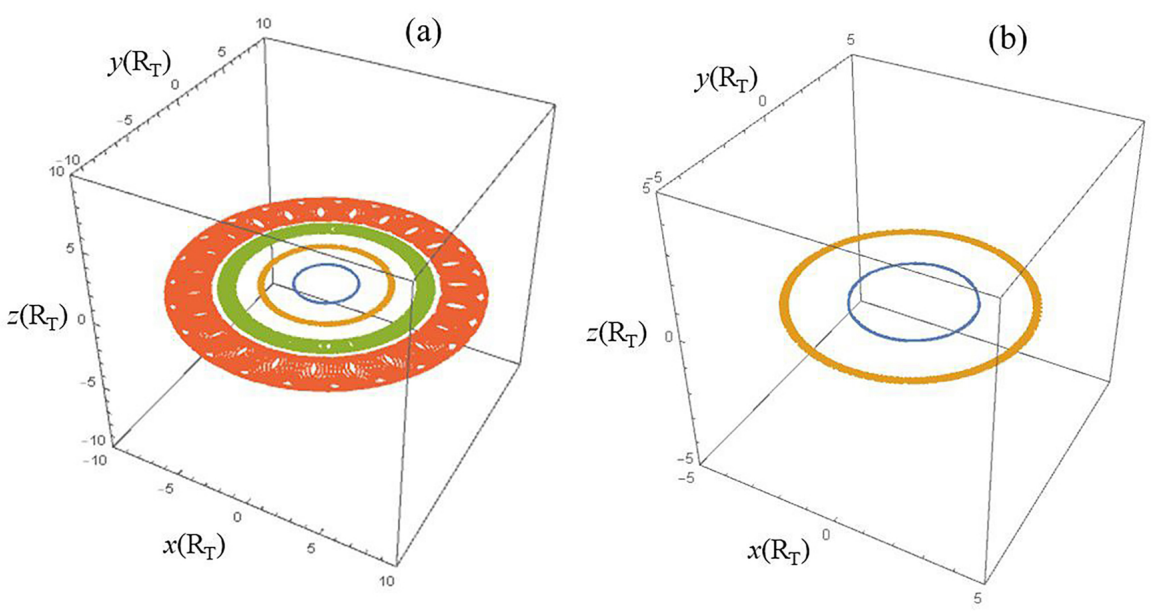

Figura 6: Efeito do vento solar nas trajetórias das cargas que se movem na região do equador. (a) Modelo de um único dipolo simples. (b) Modelo de dois dipolos com $n=20$, simulando o efeito do vento solar. As dimensões dos eixos estão em unidades do raio da Terra. 

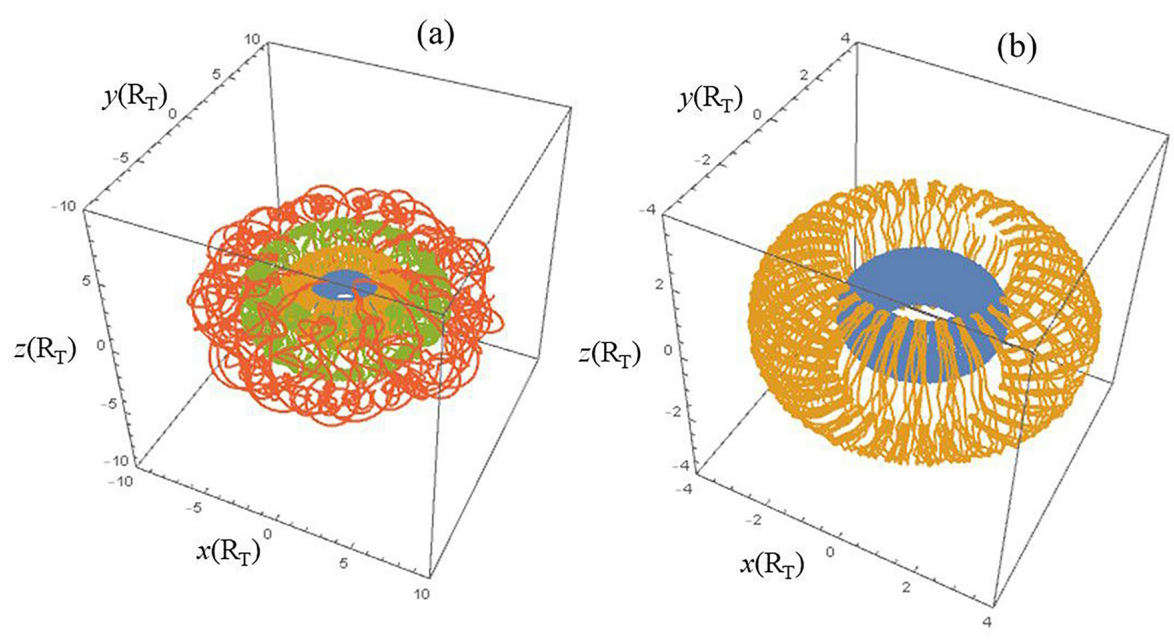

Figura 7: Efeito do vento solar na formação dos cinturões de Van Allen. (a) Modelo de um único dipolo simples. (b) Modelo de dois dipolos com $n=20$, simulando o efeito do vento solar. As dimensões dos eixos estão em unidades do raio da Terra.

os cinturões, é elegantemente compreendido por meio da introdução dos chamados invariantes adiabáticos. Para estudar a formação dos cinturões, consideramos a velocidade inicial do próton formando o ângulo $\theta=60^{\circ}$ com a direção do equador, posicionado inicialmente na região do equador, e acompanhamos o seu movimento por 200 segundos. Na Figura 7 (a) apresentamos a formação dos cinturões no modelo de um único dipolo, representando a situação em que temos apenas o efeito do campo magnético da Terra, sem perturbação, sendo levado em conta. Este cenário prevê além dos dois cinturões mais internos, de raios médios em torno de $2 R_{T}$ (azul) e $4 R_{T}$ (amarelo), outros mais externos (verde e vermelho). Aqui, mais uma vez, consideramos partículas com diferentes posições iniciais ao longo da direção $x$ com energias cinéticas de $10 \mathrm{MeV}$ 33], gerando cada um dos quatro cinturões coloridos representados na figura. Na Figura 7 (b) expomos mais uma vez a limitação imposta por um vento solar intenso, simulado por meio de dois momentos de dipolo de razão $n=20$ e separados por uma distância $d=20 R_{T}$, em que os dois cinturões mais externos representados na Figura 7 (a) são varridos pelo vento solar.

Tanto a Figura 6 quanto a 7 demonstram a limitação na quantidade de partículas carregadas na magnetosfera, introduzida por um vento solar intenso. Tal efeito é ocasionado pela magnetopausa, fronteira que delimita a região do plasma proveniente do vento solar da região da magnetosfera. Aqui em nosso modelo tal efeito é simulado pelo enfraquecimento do campo magnético terrestre, devido à presença do dipolo magnético externo adicional $\mathbf{M}$, na região da magnetopausa (curvas vermelhas representadas na Figura 51. O argumento do enfraquecimento da magnetosfera, próxima à magnetopausa, pode ser visto como um forte candidato para explicar o fato de apenas três cinturões de cargas terem sido observados até hoje, sendo o terceiro mais externo tendo sido observado há apenas alguns anos atrás por satélites da missão Van Allen Probes da NASA [34].

\section{Considerações Finais}

Neste trabalho propomos uma forma de abordar e discutir a formação dos cinturões de Van Allen em uma aula de eletricidade e magnetismo, em cursos de nível superior em ciências e engenharias. Demonstramos que tal abordagem pode ser feita mediante a aplicação básica da segunda lei de Newton e da força de Lorentz que atua em uma partícula carregada em movimento. Além disso discutimos uma forma de abordar a origem do campo magnético da Terra por meio da teoria do dínamo, que de maneira simples e pictórica pode ser vista e pensada em termos do campo magnético gerado por uma corrente circulante em um anel, o qual associamos ao campo de um dipolo magnético. Apresentamos também dois cenários para a magnetosfera terrestre e como modelá-la, de maneira simples, na ausência (Figura 3) e na presença de um vento solar (Figura 5), descrito em termos dos campos de dois dipólos magnéticos superpostos. Por fim discutimos aspectos relacionados à formação dos cinturões de Van Allen e dos anéis de cargas em movimento que envolvem a Terra, em que mostramos uma maneira de abordar a limitação na quantidade desses anéis e cinturões por meio do modelo de dois dipólos magnéticos, utilizado para simular a deformação da magnetosfera terrestre, promovida pela ação do vento solar. Argumentamos que essa limitação surge devido ao processo de varrimento dos anéis e cinturões mais externos, pelo vento solar intenso, em decorrência do limite da magnetosfera terrestre imposta pela magnetopausa.

\section{Referências}

[1] S.L. Thompson, Petrus Peregrinus de Mariocurt and his 
Epistola de Magnete (Oxford University Press, London, 1907).

[2] W. Gilbert, De Magnete, Magneticisque Corporibus, et de Magno Magnete Tellure (Peter Short, London, 1600).

[3] H.C. Ørsted, Selected Scientific Works of Hans Christian Ørsted (Princeton University Press, Nova Jersey, 2014).

[4] R.T. Merrill e M.W. Mcelhinny, The Earth's magnetic field: Its history, origin and planetary perspective (Academic Press, London, 1983).

[5] E.Echer, Revista Brasileira de Ensino de Física 32, 1 (2010).

[6] T.W. Lezniak e J.R. Winckler, Journal of Geophysical Research 75, 7075 (1970).

[7] S.E. DeForest e C.E. McIlwain, Journal of Geophysical Research 76, 3587 (1971).

[8] D.T. Welling e A.J. Ridley, Journal of Geophysical Research: Space Physics 115, 1 (2010).

[9] E. Echer, M.V. Alves e W.D. Gonzalez, Revista Brasileira de Ensino de Física 28, 51 (2006).

[10] J.Geiss, F.Buehler, H.Cerutti, P.Eberhardt e C.Filleux, Apollo 16: Preliminary Science Report (National Aeronautics \& Space Administration, Washington, 1972).

[11] S. Chapman e V.C.A. Ferraro, Nature 126, 3169 (1930).

[12] S. Chapman e V.C.A. Ferraro, Terrestrial Magnetism 36, 77 (1931).

[13] S. Chapman e V.C.A. Ferraro, Terrestrial Magnetism 38, 79 (1933).

[14] J.A. Van Allen, Journal of Jet Propulsion 28, 588 (1958).

[15] S.M. Krimigis e J.A. Van Allen, Journal of Geophysical Research 72, 5779 (1967).

[16] E. Costa Jr, F.J.R. Simoes Jr, F.R. Cardoso e M.V. Alves, Rev. Bras. Ensino Fís. 33, 4301 (2011).

[17] D.N. Baker, I.A. Daglis e V. Bothmer, in: Space WeatherPhysics and Effects, (Springer, Berlin, 2007), p. 173.

[18] R.A Freedman e H.D. Young, Física III - Eletromagnetismo (Pearson Universidades, São Paulo, 2016), p. 230.

[19] D. Halliday, R. Resnick e J. Walker, Fundamentos de Física - Eletromagnetismo (Livros Técnicos e Científicos Editora, Rio De Janeiro, 2010), p.332.

[20] M. Bezerra, W.J.M. Kort-Kamp, M.V. Cougo Pinto e C. Farina, European Journal of Physics 33, 1313 (2012).

[21] M. Korte, C.G. Constable, Earth and Planetary Science Letters 236, 348 (2005).

[22] W.F. Mcdnough, Treatise on geochemistry 2, 547 (2003).

[23] W.F. Mcdnough, Earth's core. Encyclopedia of geochemistry. Marshall, CP and Fairbridge, RW (Editors), Kluwer Academic Publishers, Dordrecht, p. 151-156, (1998).

[24] J. A. Ratcliffe, An introduction to ionosphere and magnetosphere (Cambridge University Press, São Paulo, 1972).

[25] E. W. Hones, Journal of Geophysical Research 68, 1209 (1963).

[26] K.Watanabe e T. Sato, Journal of Geophysical Research: Space Physics 95, 75 (1990).

[27] S. Chapman e J. Bartels. Geomagnetism, Analysis and Physical Interpretation of the Phenomena (Oxford Univ. Press. Reino Unido, 1940).

[28] J.G. Lyon, Science 288, 1987 (2000).

[29] D.M. Oliveira e M.V.D. Silveira, Revista Brasileira de Ensino de Física 38, 1 (2016).
[30] V. A. Chobotov, Orbital mechanics (American Institute of Aeronautics and Astronautics, Reston, 2002).

[31] J. E. García Farieta e A. Hurtado, Revista Mexicana de Física E. 65, 64 (2019).

[32] M.K. Öztürk, American Journal of Physics 80, 420 (2012).

[33] J. Torsti, A. Anttila, L. Kocharov, P. Mäkelä, E. Riihonen, T. Sahla M. Teittinen, E. Valtonen, T. Laitinen e R. Vainio, Energetic Geophysical research letters 25, 2525 (1998).

[34] D. N. Baker, S. G., Kanekal, V. C. Hoxie, M. G., Li, X. Henderson, H. E. Spence, S. R. Elkington, R. H. W. Friedel, J. Goldstein, M. K. Hudson, G. D. Reeves et al., Science 340186 (2013). 